\title{
Taxonomy and medical aspects of amoebae
}

Pathogenic and Non-Pathogenic Amoebae. By B. N. Singh. Pp. ix + 235. (Macmillan: London and Basingstoke, October 1975.) $£ 15$.

DR SINGH's book is divided into two sections-'Aerobic' and 'Anaerobic'. 'Anaerobic amoebae' is devoted to a full and useful review of work (much of it carried out in India) on the cultural requirements and pathogenicity of the dysentery amoeba. The problem of encystment is dwelt on, and methods of killing the cysts while still within the patient are discussed. Encystment and excystment factors are examined, mainly by using aerobic amoebae as model organisms. 'Aerobic amoebae' covers the amoebae of soil and water, and types of particular interest, taxonomically and medically are described in detail.

Confusion may arise in many minds when the taxonomic assignments of Singh and of the occidental authority, F. C. Page, are compared. Page considers, justifiably, I think, that Singh's Hartmannella comprises the two genera Hartmannella and Acanthamoeba. All pathogenic strains of Hartmannella (Singh) so far isolated would fit into Page's Acanthamoeba. There is a further conflict between Singh and Page over the existence of the genus Didascalus which Page would incorporate in the genus Naegleria. Didascalus as described by Singh has a 'promitotic' cell division without interzonal body formation, and is capable, with some difficulty, of producing a flagellate stage. Naegleria, according to Singh, invariably produces interzonal bodies during nuclear division; but, according to Page, this is not a consistent character. In the reviewer's experience, although the 'promitotic' division of Naegleria does not invariably reveal interzonal bodies, it generally does, and it seems sensible to separate Didascalus which never does, from Naegleria which generally has the interzonal bodies and is readily capable of forming flagellates.

A third point of conflict is linked to the preceding one, and is concerned with the nomenclature of amoebae with 'promitotic' division which do not produce a flagellate stage. Decision on this point is much more confusing, as it depends on interpretation of old descriptions in the literature, which are not even certainly of pure cultures. It is however, sufficient to say that the genus termed Schizopyrenus by Singh is in all respects that termed Vahlkampfia by Page.

There is a fourth point in which Singh differs from what seems to be current terminology. He argues that the pathogenic HB1 strain of Naegleria he initially studied is the same species as that isolated and studied by the Australian workers (This is supported by independent work.) R. F. Carter named the Australian organism $N$. fowleri in a publication slightly predating the Indian workers' description and naming of $\mathrm{HBI}$ as N.aerobia. After a rather tortuous argument, Singh claims that N.aerobia has priority over N.fowleri.

The foregoing points of taxonomic confusion serve to emphasise the need for the objective taxonomic criteria of a biochemical and immunological nature which are in the course of emerging in this rapidly expanding field.

The book is well produced and easy to read. It should form a useful introduction to the subject for the student or the research worker.

D. C. Warhurst

\section{Immunosuppressive agents}

The Mode of Action of Immunosuppressive Agents. (North-Holland Research Monographs: Frontiers of Biology, Vol. 41.) By Jean Francois Bach. Pp. xviii + 379. (North-Holland: Amsterdam and Oxord; American Elsevier: New York, 1975). Dfl. 98; $\$ 40.95$.

This book is intended for the clinical immunologist who is involved with the use of immunosuppressive agents in man. Rather than review all the data related to immunosuppressive agents. for which there is admittecily scattered and incomplete information, the author has chosen to discuss four classes of agents in detail: corticosteroids (hydrocortisone and its derivatives), thiopurines (6-mercaptopurine and azathioprine), alkylating agents (cyclophosphamide) and anti-lymphocyte serum.

In the first chapter (general introduction) several important considerations related to immunosuppressive agents are discussed. This is a good beginning and provides the proper perspective for the subseauent chanters. The problems of definition of an immunosuppressive agent, anti-inflammatory effect, therapeutic index, drug evaluation and the experimental approaches to investigating a given drug are reasonably well covered.
The outline of the subsequent chapters are quite good and begin with the known chemistry of the agents, their pharmacology, toxicity, and so on, and proceed through a review of the effects of the agents on various aspects of the immune response, including clinical trials. Unfortunately, the promise provided by the first chapter and the logic of the outline of the subsequent chapters is not fulfilled by the text material.

Often the summarised reports represent a cataloguing of data and not enough critical evaluation is summarised by the author. The result will tend to confuse the new reader. A more serious objection is the lack at times of emphasising important findings that may have exciting implications. For example, cyclophosphamide may prevent allergic encephalomyelitis or thyroiditis but it will also produce a 'tolerant' state. The latter aspect is not discussed nor even noted.

Some of the review is quite outdated (for example, metabolism of cyclophosphamide) but more seriously there are a number of errors in reporting the literature as well as uncritical reporting. On p184, for instance, the author states that bone marrow grafts must be carried out $24 \mathrm{~h}$ after cyclophosphamide injection, because of the effects of residual cyclophosphamide on engrafted cells. This is true for the rat but certainly not true for the mouse. On p203, under the heading of tumor immunity, it is stated that cyclophosphamide may favour the development of L1210 leukaemia in C57BL mice. What is meant most certainly is that $\mathrm{L} 1210$ can be transplanted to a C57BL/6 mouse given cyclophosphamide. What does this have to do with tumour immunity, as this is an allograft!

The reference quoted is wrong and the data not accurately reported in several places in the chapter on alkylating agents.

Chapter 6 contains concluding remarks and guidelines for the clinical use of immunosuppressive agents. It is also disappointing, particularly in the choice of dosage and monitoring of the effects of a given agent on a disease process. In treating a disease process it would seem wisest to decide what clinical effects one desires and then treat the disease with doses of agents designed to give acceptable clinical toxicity. The acceptable toxicity, of course, will be dictated by the severity of the disease being treated. The author does not emphasise this philosophy but instead discusses in vitro monitoring of immunological function.

In view of the serious criticisms listed above, I cannot recommend this book to those interested in immunosuppression or immunology.

George W. Santos 